Research Paper

\title{
Integrated analysis of dosage effect IncRNAs in lung adenocarcinoma based on comprehensive network
}

\author{
Yunzhen Wei ${ }^{1, *}$, Zichuang Yan ${ }^{1, *}$, Cheng $\mathrm{Wu}^{1}{ }^{1}$, Qiang Zhang ${ }^{1}$, Yinling Zhu ${ }^{1}$, Kun $\mathrm{Li}^{1}$ \\ and $Y a n X^{1}$ \\ ${ }^{1}$ College of Bioinformatics Science and Technology, Harbin Medical University, Harbin, China \\ * These authors have contributed equally to this work \\ Correspondence to: Yan Xu, email: xuyan@ems.hrbmu.edu.cn \\ Keywords: SCNA, dosage effect, IncRNAs, comprehensive network, lung adenocarcinoma \\ Received: June 14, $2017 \quad$ Accepted: July 25, $2017 \quad$ Published: August 03, 2017
}

Copyright: Wei et al. This is an open-access article distributed under the terms of the Creative Commons Attribution License 3.0 (CC BY 3.0), which permits unrestricted use, distribution, and reproduction in any medium, provided the original author and source are credited.

\section{ABSTRACT}

Accumulating evidences indicate that cancer-related IncRNAs occur frequent somatic copy number alternation (SCNA). Although individual SCNA IncRNAs have been implicated in tumor biology, their regulatory mechanism has not been assessed in a systematic way. In order to explore the expression characteristics and biological functions of SCNA IncRNAs in cancer, we built a computational framework based on IncRNA expression profiles, IncRNA copy numbers and dosage sensitivity score (DSS). First, we found that the IncRNAs with different DSS were involved in distinct biological processes, while those with the same DSS had similar functions. Second, some of the IncRNAs participated in the progression and metastasis of lung adenocarcinoma (LUAD) through cis-acting regulation. In IncRNA-TF-mRNA network, IncRNAs interacted with 4 TFs and affected the immune system, and further influenced LUAD progression. Third, competing endogenous RNA network analysis inferred that InCRNA ENSG00000240990 competed with HOXA10 to absorb hsa-let-7a/b/f/g-5p and affected patient prognosis in LUAD. Last but not least, by integrating target information of miRNA we also provided a new perspective for the discovery of potential small molecule drugs. In summary, we systematically analyzed the regulatory role of SCNA IncRNAs. This work may facilitate cancer research and serve as the basis for future efforts to understand the role of SCNA IncRNAs, develop novel biomarkers and improve knowledge of tumor biology.

\section{INTRODUCTION}

Non-small cell lung cancer (NSCLC), which accounts for 70-80 percent of lung cancer, is one of the leading causes of death world-wide and is notoriously difficult to treat effectively. Particularly, lung adenocarcinoma (LUAD) is the most common subtype of NSCLC $[1,2]$. The success of cancer treatment depends on the early examination and diagnosis. However, many patients are diagnosed with cancer in metastasis or terminal period. Though the technology of molecular diagnosis and target therapy is improving, the overall survival of LUAD patients in 5 years is low [3]. Therefore, it is imperative for clinic to identify suitable molecular markers for LUAD diagnosis and prognosis.
The high-throughput sequencing analysis revealed that the non-coding RNA made up the preponderance of genome and one of the subsets, the lncRNA, was proved to affect tumor development and pathology through chromosome modification, transcription and post-transcriptional process [4]. For example, IncRNA MALAT1 regulated the expression of genes that were associated with lung cancer metastasis [2]; IncRNA CCAT1 acted as an oncogene and promoted chemoresistance in docetaxel-resistant LUAD cells [1]. Recently, a long intergenic non-coding RNA (lincRNA) p21 was identified as a mediator and a key target of p53 DNA damage response $[5,6]$.

Furthermore, cancer is a kind of genetic disease that is related with multiple variations of genome. Davoli et al. found that the cancer driver genes tended to have SCNA 
in genome, and the frequent alterations of DNA copy number promoted cancer progression [7]. Actually, SCNA was verified to be associated with various cancers such as LUAD, breast cancer and liver cancer [8-10].

In fact, SCNA affects cancer progression in an indirect way and contributes to pathway disorder by regulating gene expression. Previous studies showed that the dosage sensibility of SCNA was closely related to cancer [11]. The aberrance of gene expression that induced by SCNA could be described by dosage sensibility [12]. Nevertheless, because of the difference of dosage sensibility, SCNA may not have the same influence on gene expression. The change of dosage sensibility could control the disorder of gene expression. Moreover, some studies indicated that SCNA was a key mechanism for the disorder of cancer-related lncRNAs [13]. For example, PVT1 was found to occur copy number amplifications accompanied by oncogene MYC and promoted the expression of MYC. The MYC expression may return to the precancerous condition through breaking the cooperative relationship between PVT1 and MYC [14]. Evidently, the discovery supported the hypothesis that the dosage effect of lncRNAs had potential functions in cancer. Therefore, it is important to have a deep insight into the lncRNAs that occur SCNA to explain the potential mechanism of disease progression and find accurate treatment of cancer.

Nowadays, only a small group of lncRNAs is known to be related with diseases. Though lacking the ability to code proteins, IncRNAs can regulate the transcription and translation of neighboring genes significantly, leading to a series of biological progressions such as dosage compensation, genomic imprinting and cell cycle control [15]. Typically, many studies described the function of IncRNAs based on the Guilty by Association principle [16]. These studies explored the functions of lncRNAs in biological progression and cancer by constructing a co-expression model of protein-coding genes (PCGs) and $\operatorname{lncRNAs}[16,17]$. For instance, lncRNA MALAT1 participated in LUAD metastasis via regulating gene expression [2]. Consequently, with the burning desire to describe the functions of lncRNAs deeply, more and more regulated functions were discovered. First, IncRNAs can regulate gene expression through cis-regulation and transregulation function $[18,19]$. With cis-regulation function, lncRNAs may influence the expression of neighboring genes [20]. Accumulated evidences demonstrated that part of lncRNAs were positively correlated with the expression level of PCGs [17, 21]. For example, the lncRNA Jpx positively affected the expression of neighboring gene Xist [22]. In addition, recent studies also found that lncRNA was related with TF [23]. Take lncRNA Evf-2 as an example, it could promote the transcription of Dlx gene cluster by forming a stable compound with TF Dlx2 [24]. A novel hypothesis believed that IncRNAs could act as miRNA sponges and regulate gene expression through competing endogenous RNA (ceRNA) mechanism [1, 25]. An increasing number of evidences proved that ceRNA lncRNAs played a key role in cancer occurrence and progression. Xu et al. found that the majority of the interactions of miRNA sponges was cancer-specific which formed conserved and cancer-specific models by the interactions among sponges [26]. Nonetheless, seldom do people systemically describe the function and expression characteristics of SCNA lncRNAs. Our study analyzed the genomic characteristics of SCNA lncRNAs based on DSS. Furthermore, we described the functions and regulations of SCNA lncRNAs in cis-regulating network, transregulating network and ceRNA network. We constructed an IncRNA-function network and depicted the functions of SCNA lncRNAs in cancer progression. Finally, we identified prognosis-related lncRNAs and nominated potential small molecular drugs for LUAD treatment. Taken together, our study systematically analyzed SCNA lncRNAs, provided a foundation for deeper understanding the role of cancer-related SCNA lncRNAs and improved knowledge of tumor biology.

\section{RESULTS}

\section{LncRNAs exhibit frequent SCNAs in LUAD}

We proposed a pipeline to systemically analyze the functions of SCNA IncRNAs, and the approach was applied to the LUAD dataset (Figure 1). We analyzed the LUAD copy number profile that was calculated by GISTIC2.0 and identified 128 independent genomic variable fragments (49 gains and 79 losses). Previous study indicated that SCNA was an important mechanism that led to the dysregulation of lncRNAs in cancer, especially for those cancer types whose genomes contained abundant SCNAs [27]. Therefore, we obtained the genomic local information of 13,870 lncRNAs from GENCODE. In order to nominate the lncRNAs that acted as driver genes in tumor occurrence and development, the lncRNAs sites were mapped to the 128 independent genomic variable fragments (Supplementary Figure 1). 429 and $2,923 \operatorname{lncRNAs}$ were mapped to the focal gains and losses fragments, respectively. In total, 1,060 lncRNAs had expression value (179 amplified and 881 deleted lncRNAs, Supplementary Data S1).

\section{The dosage effect score of IncRNAs}

We calculated the copy number frequency of each IncRNA in variable fragments through copy number discretization matrix. The alteration that occurred in at least $20 \%$ samples was defined as high-frequency alteration. In fact, few lncRNAs occurred high-frequency gain and loss at the same time. As a result, 3,499 lncRNAs 


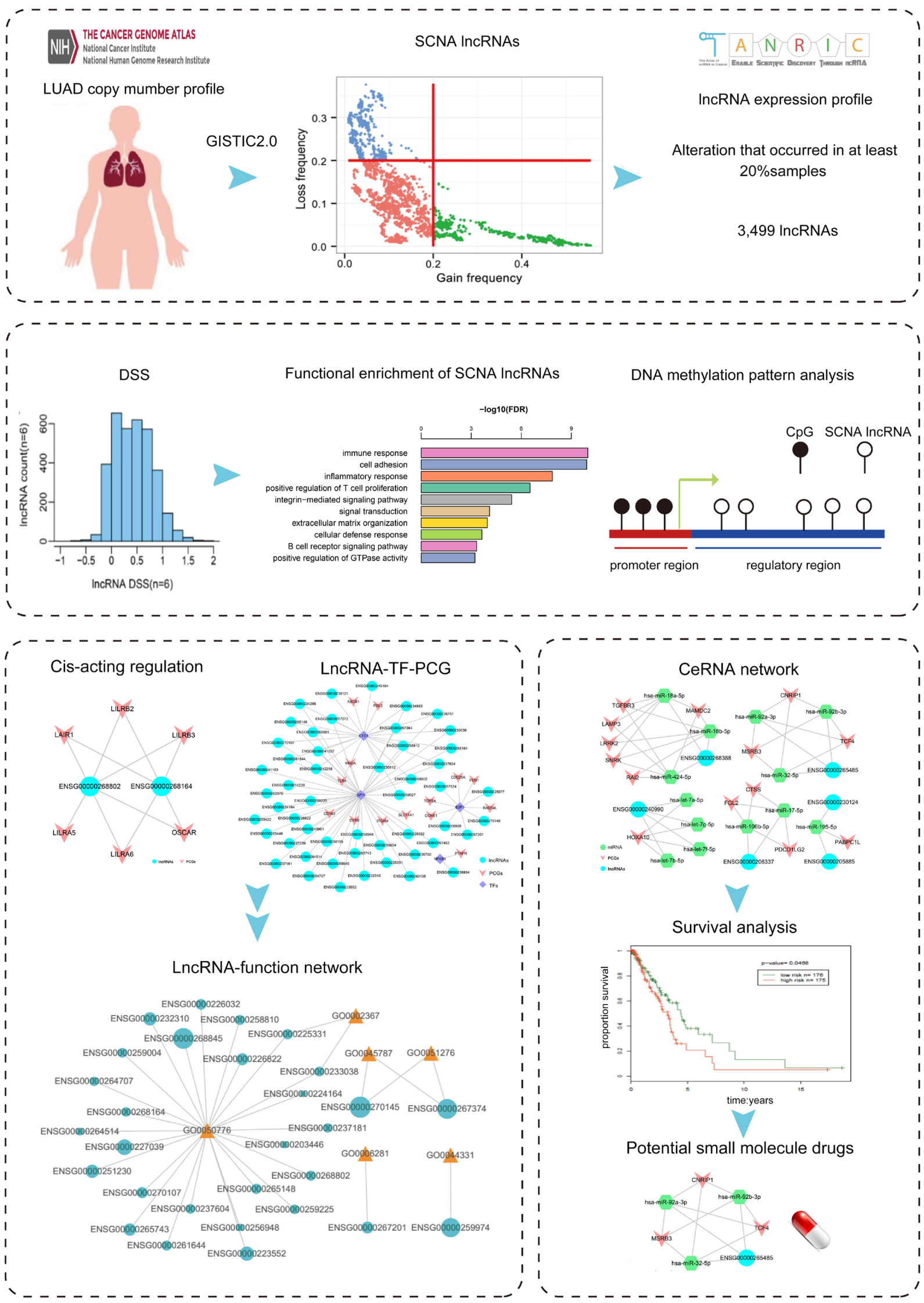

Figure 1: Flowchart of the study. A. The identification of SCNA lncRNAs. B. The calculation of DSS of SCNA lncRNAs and the basic analysis. C. and D. The construction of cis-acting network, lncRNA-TF-PCG network and ceRNA network. 
had high-frequency alterations, most of which were intergenic and trans-lncRNAs (Supplementary Data S2).

In order to evaluate the impact of SCNA on the deregulation of lncRNAs, we analyzed the association between lncRNA copy number and RNA expression level by calculating DSS [28]. The DSS of lncRNAs was showed in Figure 2A. The result showed that most scores of IncRNAs were low.

In the DSS calculation formula, the parameter $n$ determined the monotonicity score. In order to obtain precise result, we performed parameter selection. The initial parameter $\mathrm{n}$ was set as 6 . As control, the $n=5,7$ and 15 were selected to calculate the DSS of genes (Figure 2B-2D). The distributions of DSS were similar in different parameters, confirming the robust of our approach.

\section{Enrichment analysis of SCNA IncRNAs}

As we know, most of the lncRNAs lack annotation. Their functions are usually assessed by the co-expressed PCGs. Our study identified significant 25,871 lncRNA-

A

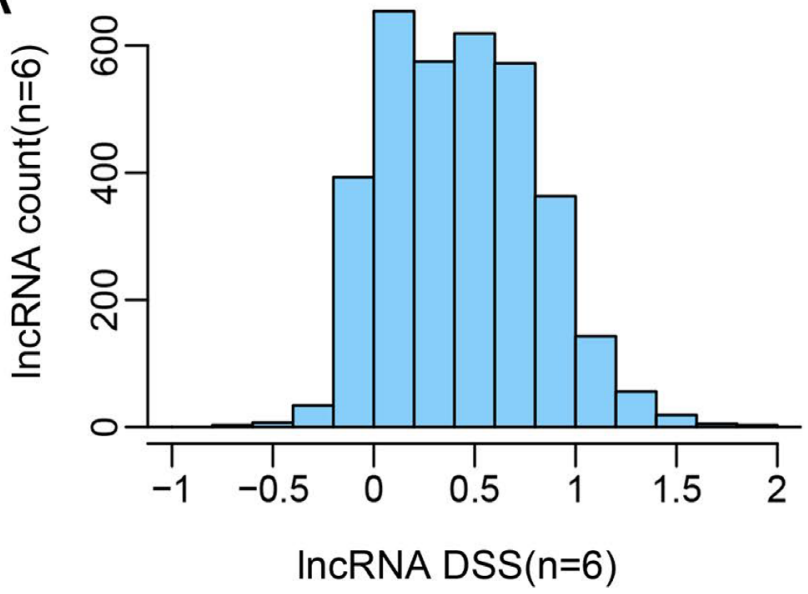

C

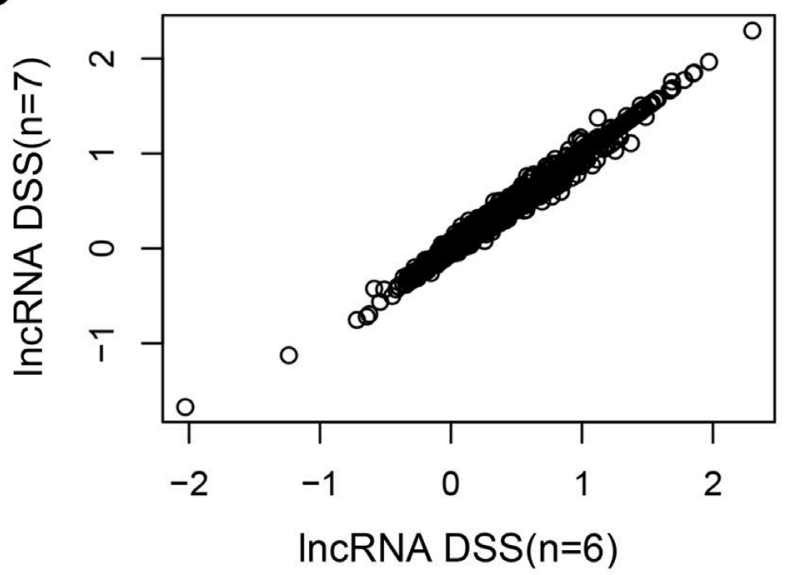

PCG pairs (1,133 lncRNAs and 1,976 PCGs) based on the profile of 3,449 SCNA lncRNAs and 3,654 differentially expressed PCGs. We divided 1,133 lncRNAs into 7 groups based on the DSS of IncRNAs and performed functional enrichment analysis for co-expression PCGs of each group $($ FDR $<0.05)$.

The result indicated that the low-sensitivity lncRNAs were mainly enriched in immune-associate biology processes, including immunoreaction, inflammatory response, cell adhesion, chemotaxis, cell proliferation, repair in trauma, angiogenesis and cell migration (Figure 3). The immune system was an important mechanism for immune response and had positive effects on immunological surveillance, defense and regulatory. Immune system could hinder the escape of cancer cell by specific mechanism. Lung cancer immunization therapy located the target site of cancer cells by using immune checkpoint inhibitors, which contributed to the interruption of misleading signals that emitted by cancer cell and the elimination of cancer cells $[29,30]$. Researchers could re-activate the immune cell and inhibit the growth of cancer cell by breaking through

B

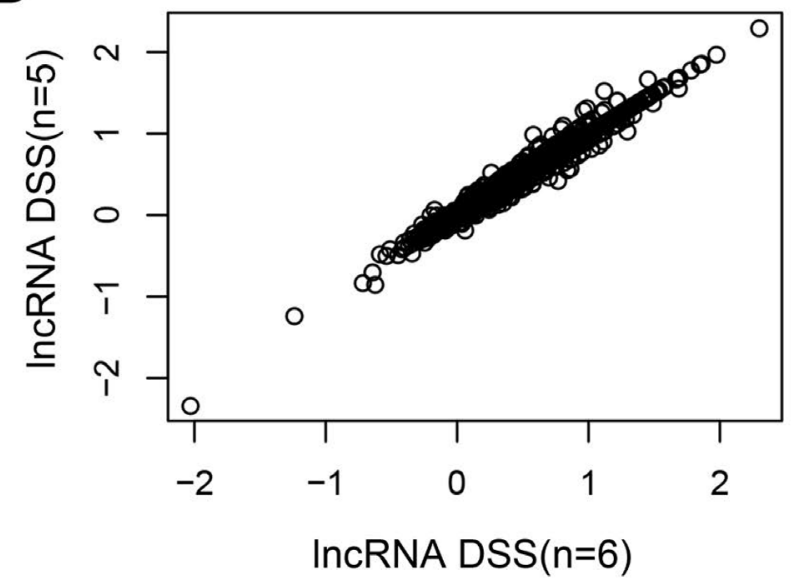

$D$

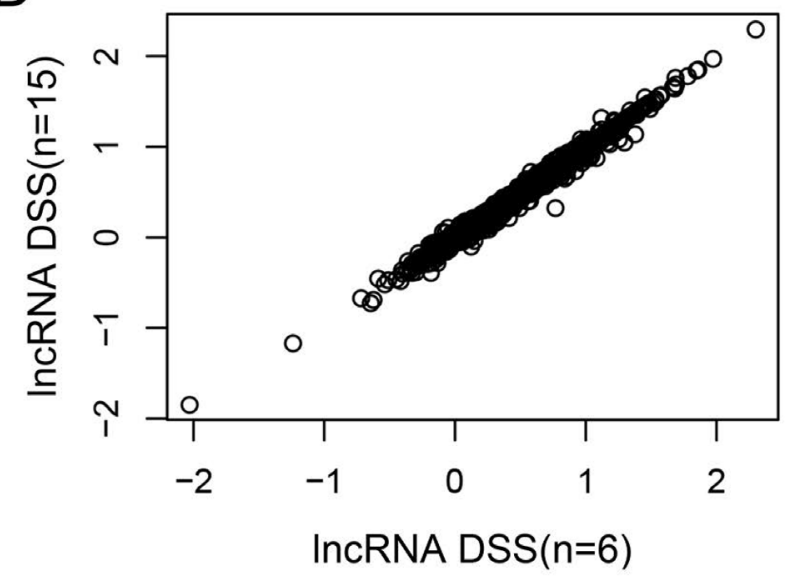

Figure 2: The DSS of IncRNAs in different parameters. A. The DSS of $\operatorname{lncRNAs}(n=6)$. X-axis represents the DSS of each lncRNA, y-axis represents the number of lncRNAs. B.-D. The comparison of DSS among different parameters $(n=5,6,7$ and 15$)$. 
the sentinel point. The low-dosage sensitive lncRNAs that identified by our study may provide new perspective for the identification of sentinel point. The functions of SCNA lncRNAs was changed from immune system to some key processes that maintained cell ordering and genomic DNA stability, including cell cycle, cell differentiation, DNA repair, cytoskeleton and chromosome assembly. The disorder of cell cycle regulation was one of the characteristics of cancer cells [31] and the regulation of cell cycle was an elaborate equilibrium process. Nowadays, the researches of various regulatory factors and regulatory mechanisms provide a solid theory foundation for tumor occurrence and development. Therefore, the high-dosage sensitive lncRNAs that we identified may contribute to the further research of cell cycle regulatory factor, and the relationship among them could promote the understanding of oncobiology. cell cycle checkpoint
microtubule cytoskeleton organization

DNA replication

DNA metabolic process chromosome segregation

cell proliferation

microtubule-based movement

cell cycle

cell division

organelle fission

$\mathrm{M}$ phase

mitosis

nuclear division

positive regulation of immune response microtubule-based process positive regulation of immune system process positive regulation of response to stimulus blood vessel development vasculature development immune effector process regulation of lymphocyte activation
0

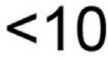

$<15$

$<20$

$<40$

$<60$
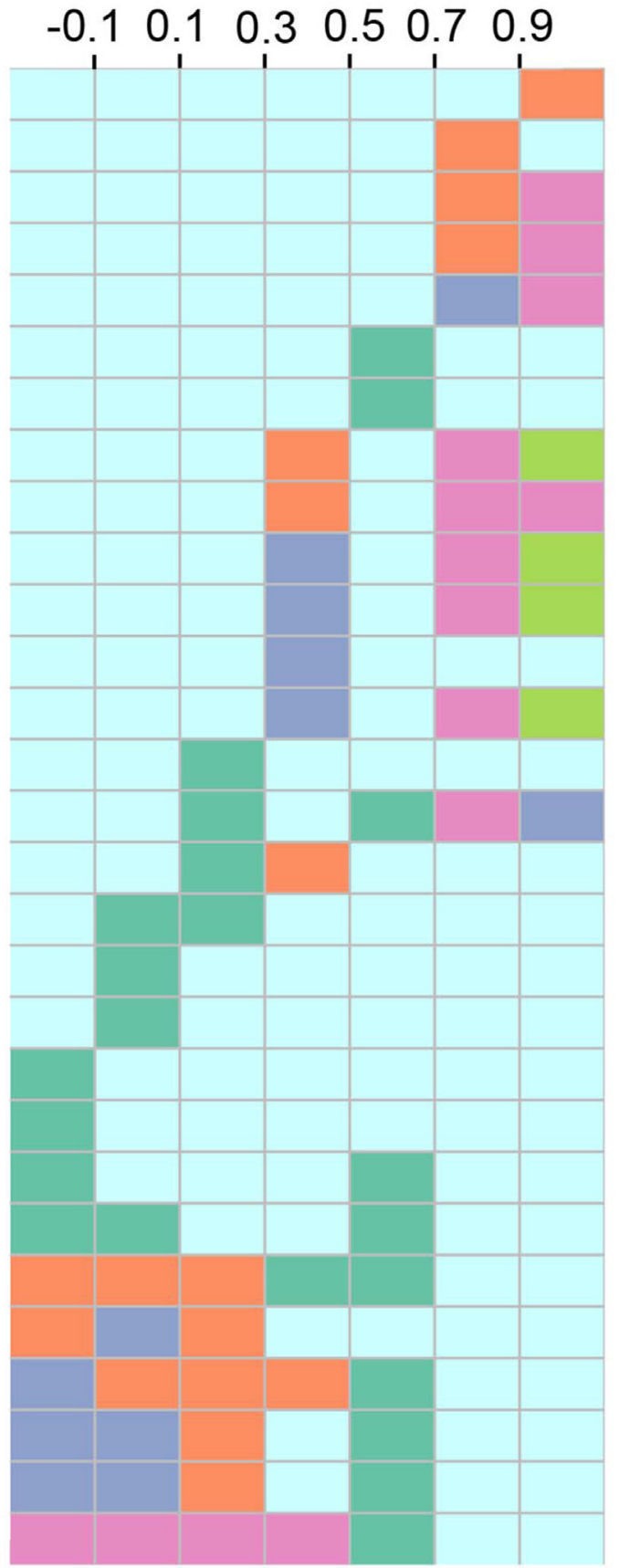

Figure 3: Functional enrichment of SCNA IncRNAs. The columns represent the score Intervals of lncRNAs. The rows represent the significant biological processes that enriched by lncRNAs. The different colors represent the degree of the enrichment significance (-log 10 (FDR)). 


\section{DNA methylation analysis}

In order to evaluate the impact of methylation on SCNA lncRNAs, we identified differentially methylated lncRNAs in promoter regions by $t$-test method [32] (Supplementary Data S3; 66 hypermethylation and 2 hypomethylation lncRNAs, respectively). 22 of 66 hypermethylation IncRNAs were expressed in LUAD. Furthermore, the expression of 22 lncRNAs was lower than that of the others in LUAD (Wilcoxon test, $p=$ 0.0022). 17 hypermethylation lncRNAs occurred SCNA at the same time and had low expression (Wilcoxon test, $p=0.015$, Figure 4). To explore the relationship between low expression and hypermethylation, we calculated PCC for each lncRNA. In total, 12 of them were negatively correlated $(p<0.05$, Supplementary Data S4). These results implied that the expression of the 12 lncRNAs was affected by both of the copy number and methylation level in promoter regions.

\section{Cis-acting network}

Regulatory lncRNAs can influence or interact with nearby or distant genes in both cis-acting regulation and trans-acting regulation $[33,34]$. We first constructed a cisacting network based on co-expression SCNA lncRNAs and differentially expressed PCGs pairs. In total, we obtained 146 pairs, including 130 SCNA lncRNAs and 128 differentially expressed PCGs (Supplementary Figure S2). The result exhibited that several lncRNAs significantly regulated multiple neighbor PCGs. One explanation for the phenomenon was that we selected differentially and significantly co-expressed PCGs. The ENSG00000268802 and ENSG00000268164 shared the same 5 PCGs (LAIR1, LILRB2, LILRB3, LILRB6 and OSCAR), and 4 of them belonged to leukocyte immunoglobulin-like receptor family (Figure 5). A study pointed out that LILRB2 was a receptor of natural killer (NK) cell [35] and was associated with lung cancer cell [36]. NK cells were the

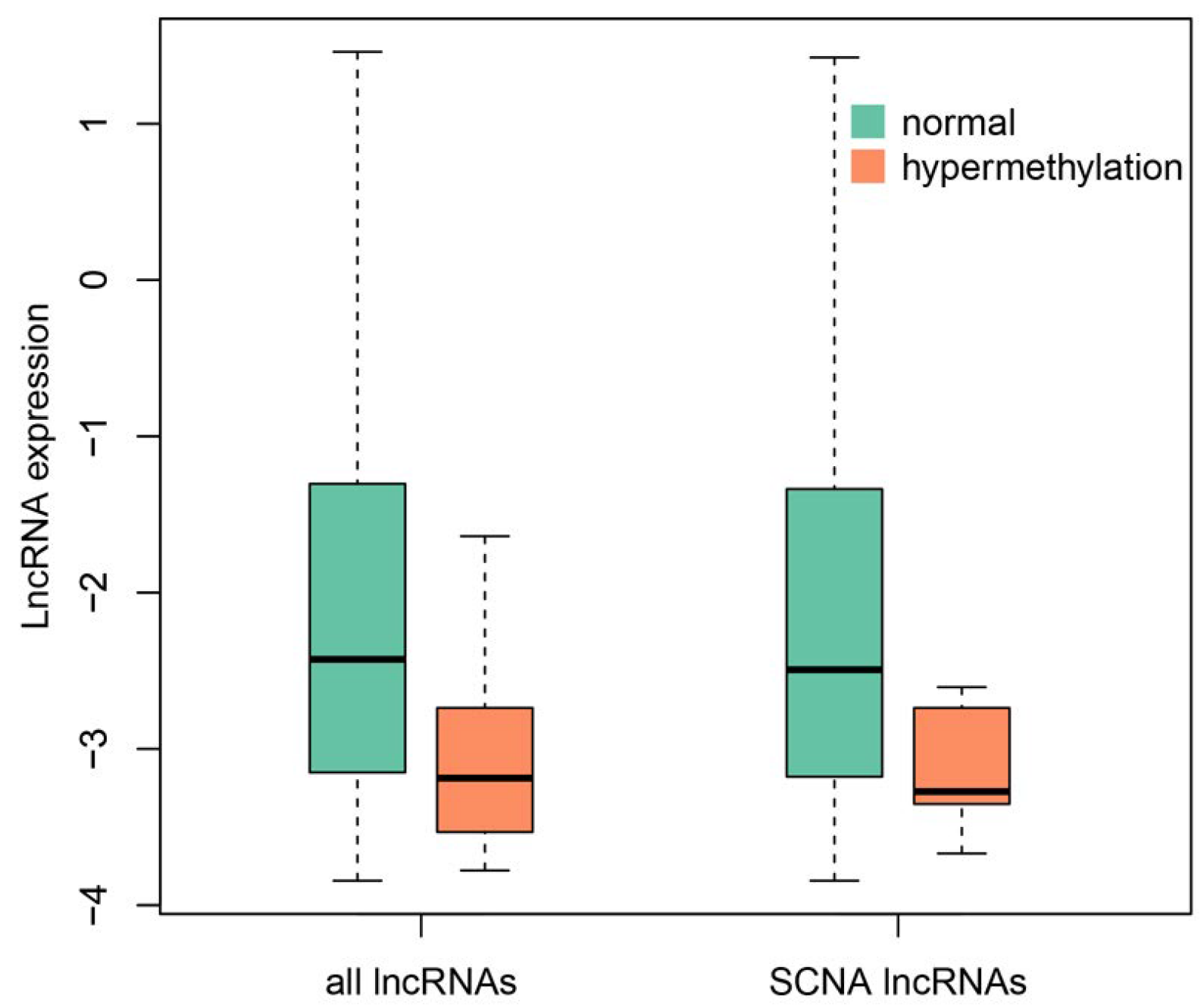

Figure 4: The expression comparison between hypermethylated IncRNAs and others. The green represents the normal IncRNAs that expressed in LUAD, the orange represents the hypermethylated lncRNAs (Wilcoxon test, $p$-value $=0.0022$ and 0.015 , respectively). 
major subset of innate lymphoid cells and the first line of defense of infection endowed with complex regulatory roles [37]. In addition, NK cells were associated with the angiogenesis of LUAD [38]. OSCAR coded osteoclastassociated immunoglobulin-like receptors and related to immune mechanism [39]. Overall, these results revealed that IncRNA ENSG00000268802 and ENSG00000268164 may function by regulating the 5 target genes in cisacting regulation. Furthermore, we found that the DSS of lncRNA ENSG00000268802 and ENSG00000268164 was low (0.03537 and -0.0188 , respectively), which further confirmed our hypothesis that the low-DSS IncRNAs mainly took part in immune-related process.

The function of lncRNAs in network could be explored by further analysis of cis-acting regulation. For example, the lncRNA ENSG00000261685 (gene symbol: RP11-401P9.4) occurred copy number deletion and down-regulated in LUAD. The result demonstrated that the nearest co-expression PCG NKD1 occurred down-regulation at the same time [40]. NKD1 was an established transcriptional target of $\beta$-catenin complexes and a marker of aberrant Wnt/b-catenin signaling [41, 42]. A study revealed that the down-regulation of NKD1 increased the invasive potential of NSCLC and correlated with a poor prognosis [43]. Moreover, the down-regulated ENSG00000259974 regulated the target PCG FOXA2 that acted as a suppressor of lung cancer in cis-acting regulation [44]. FOXA2 was a key regulator for LUAD metastasis and down-regulated in LUAD [45]. The neighboring co-expression PCG MYEOV up-regulated by the overexpression of the amplification lncRNA ENSG00000260877. MYEOV functioned as an oncogene and overexpressed in many cancers including LUAD [46]. PCG CLDN4, the neighboring co-expression gene of ENSG00000225969 up-regulated in LUAD. A research indicated that the overexpressed CLDN4 was related with LUAD carcinogenesis [47]. These results showed that the SCNA lncRNAs played an important role in the occurrence, development and metastasis of lung cancer through cis-regulating neighboring target PCGs.



Figure 5: The cis-acting subnet of SCNA IncRNAs. The blue represents the lncRNAs, the red represents the differentially expressed PCGs. 


\section{The construction of IncRNA-TF-PCG triples}

The interactions between IncRNAs and TFs can improve the expression level of their target genes [34]. We obtained 245 LUAD-related IncRNA-TF-PCG triples, including 51 lncRNAs, 4 TFs and 14 differentially expressed PCGs, respectively (FDR $<0.05$, Figure 6). 4 TFs (ETS1, SPI1, E2F1 and NFKB1) were the core of the trans-acting network and played important roles in cancer. For example, one of the TFs, ETS1, was related with the poor prognosis of LUAD [48]. Another $\mathrm{TF}$, E2F1, promoted the apoptosis and acted as tumor suppressor [49]. Especially, the target PCGs of the 4 TFs were involved in some key cancer processes according to previous studies. Take CYBB, the target PCG of TF SPI1 as an example, it was found to be related with the prognosis of LUAD [50].
Furthermore, one of the major challenges of lung cancer immunotherapy was the tumor immunogenicity and immune escape. The PCG TLR4 that identified by us took part in trans-acting triples and provided clues for the inhibition of immune escape [51]. In the trans-acting network, 35 SCNA lncRNAs regulated TLR4 via TF SPI1 and ETS1 and formed a complicated subnet. There were 33 lncRNAs that the dosage sensitivity score of which was lower than 0.3 , and $29 \operatorname{lncRNAs}$ that the dosage sensitivity score of which was lower than 0.1 (Supplementary Figure S3). Combining the enrichment result that mentioned above, we could infer that the lncRNAs which participated in trans-acting regulation may affect cancer progresses via immune system. In a word, trans-acting network was helpful for providing key insights into tumor development and immune therapy.

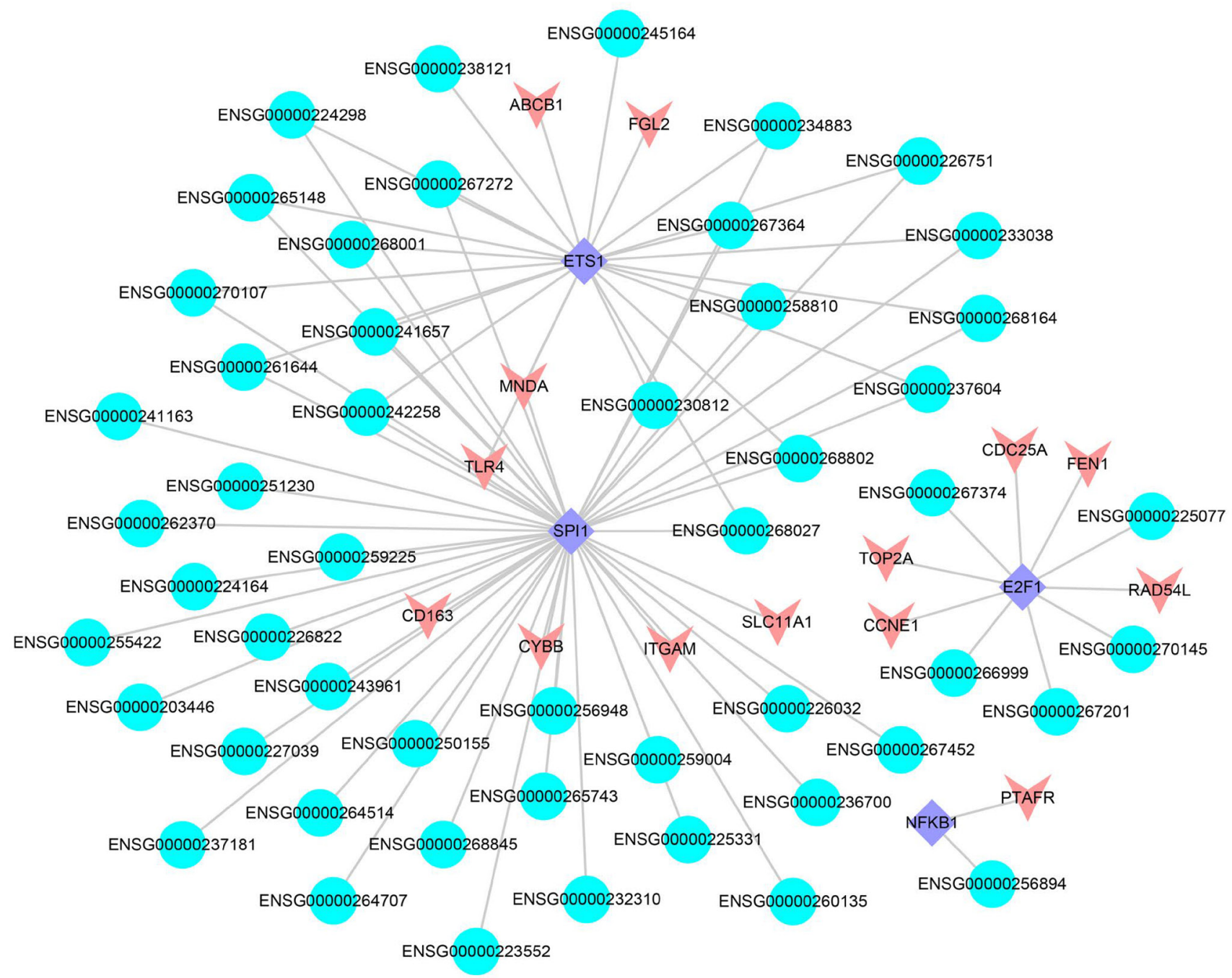

Figure 6: The IncRNA-TF-PCG network. The blue represents the lncRNAs, the red represents differentially expressed PCGs and the purple represents the TFs. 


\section{Functional prediction of IncRNAs based on IncRNA-function network}

Combining the results of cis-acting analysis and lncRNA-TF-PCG triples, we found that 10 lncRNAs involved in both cis-acting mechanism and trans-acting mechanism. It was suggested that the lncRNAs could function through different patterns. In order to provide deeply insights into the influences of SCNA lncRNAs in cancer, we obtained $171 \mathrm{lncRNAs}$ from the cis-acting analysis and lncRNA-TF-PCG triples, and searched the GO terms through their target genes. We identified the IncRNAs that was significantly enriched in GO terms by hypergeometric test and constructed lncRNAfunction network (FDR < 0.05). The network contained 29 lncRNAs and 6 GO terms (Figure 7). We found that the DSS of most of the lncRNAs that were related with immune regulation was lower than 0.5 , while the score of lncRNAs that were related with cellular process was high. For example, the DSS of the ENSG00000270145 and ENSG00000267374 was 1.016 and 0.8857 respectively, and they involved in the process of cell cycle. The results further confirmed the functions of IncRNAs with different scores. The 29 lncRNAs that were related with immunoreaction in functional network may serve as candidate targets for LUAD immunotherapy.

\section{The identification of core regulation IncRNAs via ceRNA network}

Recent studies verified that miRNAs was useful for lncRNA analysis [52-54]. For example, lncRNAs could act as miRNA sponges to regulate the expression of other transcripts [55]. In order to evaluate the ceRNA interactions that were mediated by lncRNAs, we constructed the IncRNA-miRNA-mRNA ceRNA network through a multi-step method (Supplementary Figure S4). First, we selected the PCGs that deregulated in LUAD and the lncRNAs that occurred SCNA. Second, the PCC value $(r>0.5, \mathrm{FDR}<0.05)$ was used as the strict threshold to identify significant lncRNA-PCG pairs. In addition, if both PCG and lncRNA in the same triple were co-expressed negatively with a certain common miRNA $(r<0$, FDR $<0.05)$, this triple was remained for further selection. Finally, a hypergeometric test was performed to evaluate the significance of shared miRNAs for each possible pair $($ FDR $<0.05)$.

All the functional pairs were integrated to form a miRNA-mediated lncRNA-associated ceRNA network, and we obtained 36 significant ceRNA pairs, including 6 lncRNAs, 13 miRNAs and 14 differentially expressed PCGs (Figure 8). The results showed that the lncRNAs competed with multiple PCGs to absorb miRNAs and formed a complex competition network. Take lncRNA ENSG00000268388 (FENDRR) as an example, it

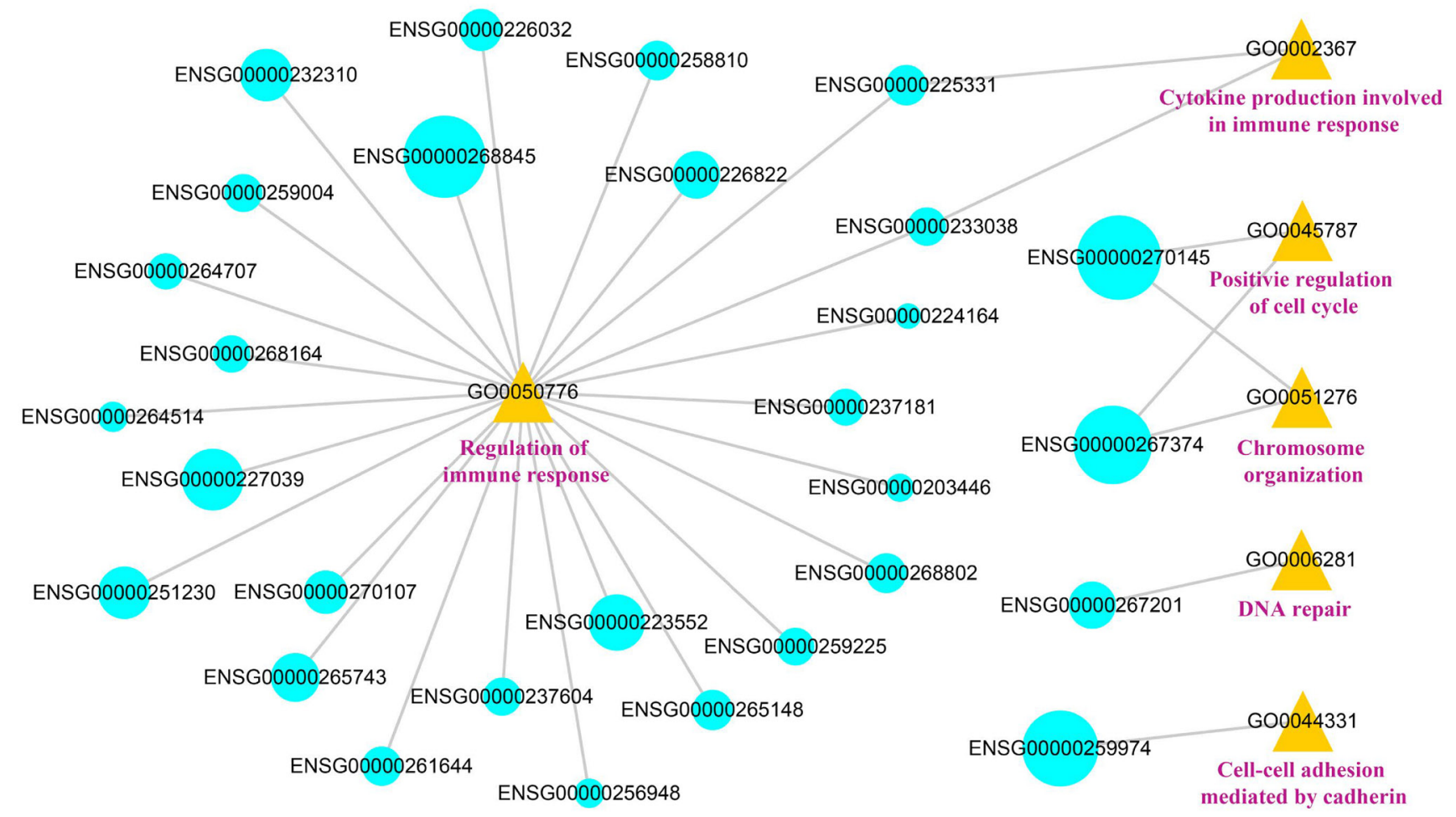

Figure 7: The visualization of the IncRNA-function network. The blue represents the lncRNAs, the yellow represents the GO number and the pink represents the GO terms. The size of the blue circles represents the DSS of the lncRNAs. 
competed with 6 PCGs to absorb 3 miRNAs and formed a complex competition subnet. It was suggested that these lncRNAs may play key roles in the regulation of cancer processes. On the other hand, the PCGs that competed with lncRNAs in these subnet also had important functions. For example, PCG CTSS, FGL2 and PDCD1LG2 (PDL2) competed with ENSG00000206337 (HCP5) and formed a subnet. Previous study indicated that FGL2 may serve as a therapeutic target or a biomarker for tumor early detection [56]. Chen et al. found that PDL2 was an immune checkpoint gene [57]. In addition, a study pointed out that the lung cancer risk sites were significantly enriched in lncRNA HCP5 [58]. It was suggested that HCP5 may involve in the process of lung cancer by competing with PDL2 and FGL2.

\section{The prognosis ability of the ceRNA network}

We calculated the risk score [59] for each lncRNAmiRNA-PCG triple based on the risk score of single factor regression analysis of each node, and divided patient samples into high-risk and low-risk groups by median. The result showed that the ENSG00000240990-HOXA10 pair interacted with 4 miRNAs (hsa-let-7a/b/f/g-5p) and was significantly associated with prognosis $(p<0.05$, Figure $9)$. The high-risk group had poor prognosis. Furthermore, the risk parameters of the 4 miRNAs were negative, while the risk parameters of the lncRNA ENSG00000240990 (HOXA11-AS) and PCG HOXA10 were positive. It was suggested that the patient survival can be affected by the ceRNA pairs. HOXA11-AS was a highly conserved lncRNA that located in the chromosome 7p15.2 [60]. Pervious study indicated that HOXA11-AS could serve as a biomarker for lung cancer metastasis and poor prognosis [61]. In our study, IncRNA HOXA11-AS and PCG HOXA10 were highly expressed (fold-change $=1.641$ and 4.683, respectively). A study demonstrated that PCG HOXA10 overexpressed in lung cancer frequently [62] and was involved in the pathogenesis of lung cancer [63]. For lncRNA HOXA11-AS, it overexpressed in LUAD and was involved in the processes of NSCLC through regulating its target genes [64].
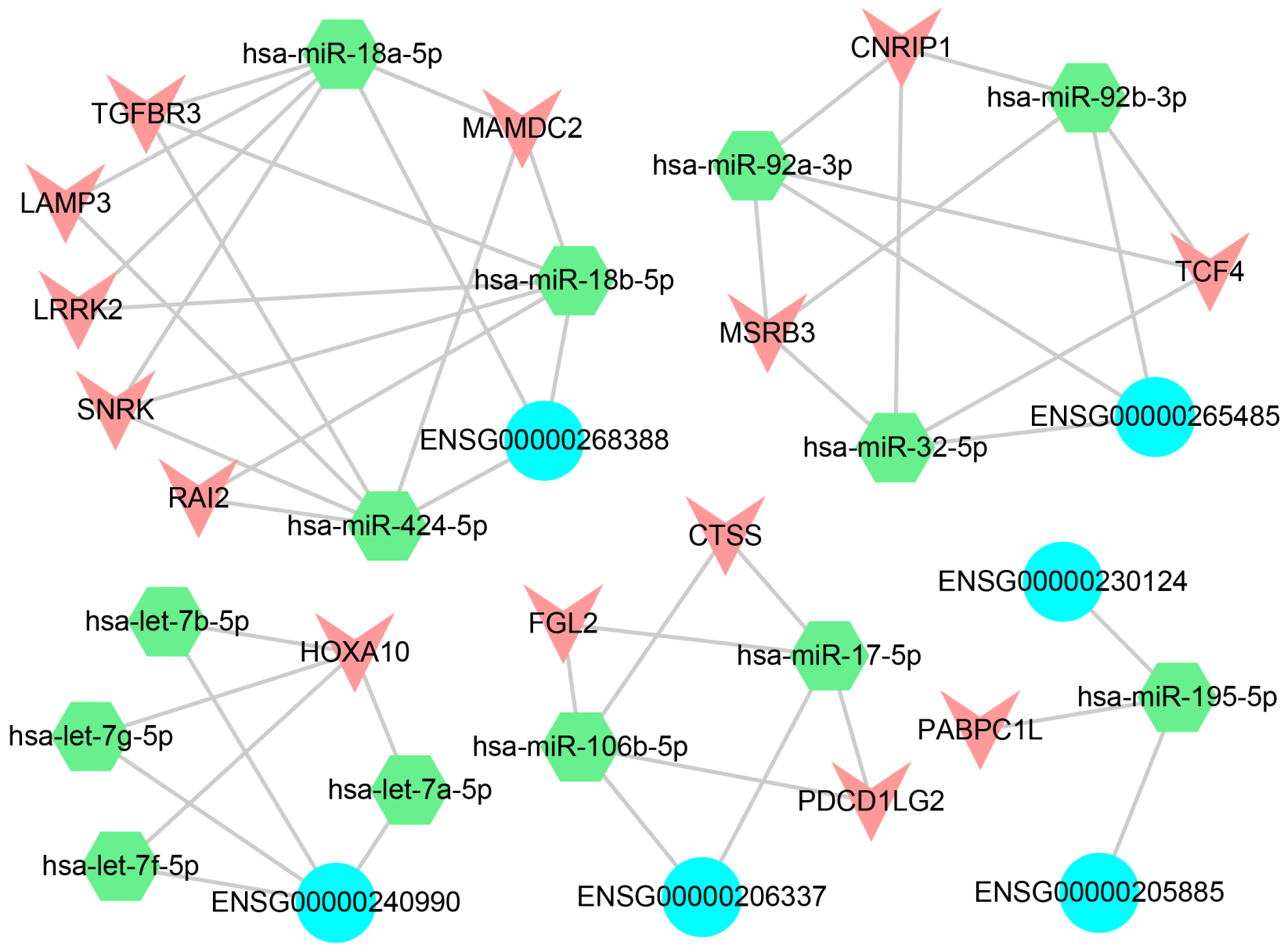

Figure 8: ceRNA network. The blue represents the lncRNAs, the green represents the miRNAs and the red represents the differentially expressed PCGs. 


\section{Potential small molecule drugs for LUAD treatment}

In the ceRNA network, the perturbation of miRNA expression can influence the expression level of lncRNAs and PCGs $[65,66]$. Therefore, we combined the information that was provided by SM2miR [67] with the module to infer potential small molecule drugs for LUAD treatment. In the ceRNA module, some potential drugs could up-regulate the hsa-let-7a/b/f/g-5p expression and further down-regulate the expression of related lncRNAs/ PCGs and contribute to the treatment of LUAD (Figure
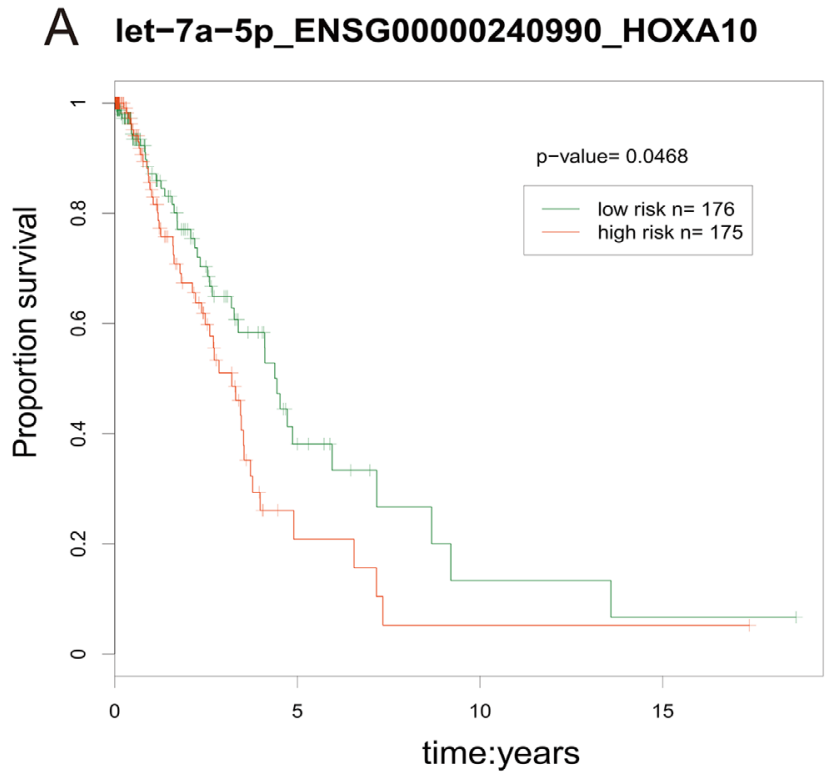

C let-7f-5p_ENSG00000240990_HOXA10

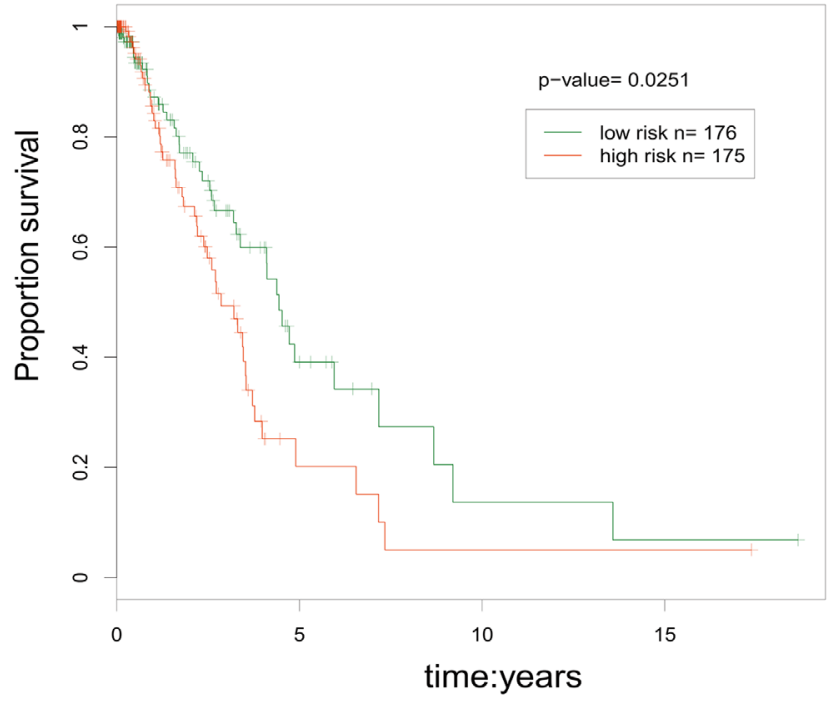

10). For example, curcumin could induce the apoptosis of cell NCI-H460 in NSCLC [68] and inhibit the transfer and invasion of lung cancer cell $[69,70]$. In LUAD A549 cell, arsenic trioxide could induce the death of apoptotic cell [70]. Metformin could inhibit the growth of LUAD cell via apoptosis-inducing [71] and improve the survival of NSCLC patients in the stage IV [72]. In addition, we found other small molecule drugs that could regulate the 3 miRNAs such as CDF (analogues of curcumin). The effectiveness of these small molecule drugs could be validated by further experiment (Supplementary Data S5).



D let-7g-5p_ENSG00000240990_HOXA10

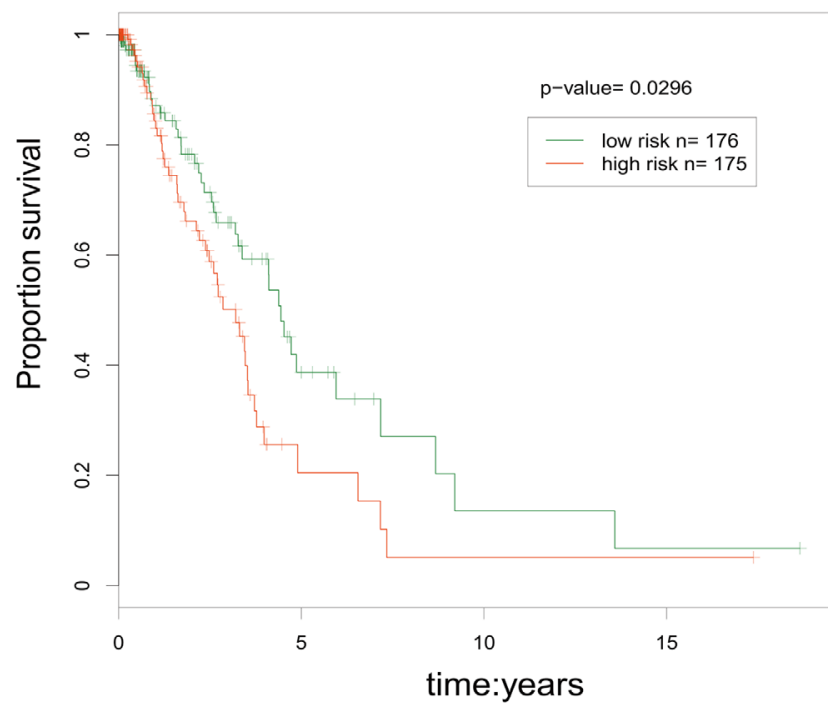

Figure 9: The prognosis-related ceRNA pairs. The red line represents high-risk groups and the green line represents the low-risk groups. Samples with low risk were found to have longer survival. 


\section{DISCUSSION}

According to the central dogma, the aberrance of copy number may affect cell functions. However, the SCNA could not directly function on cancer. It contributed to the pathway dysfunction by regulating gene expression [12]. Previous study demonstrated that SCNA was one of the most important mechanisms for IncRNA deregulation [13]. In order to discover the expression pattern of IncRNAs that affected by SCNA, we highlighted the differences of lncRNAs through matching SCNA profile and lncRNA profile, then selected dosage effect lncRNAs and analyzed the functions of lncRNAs with different DSS.

Our method that calculated DSS could well response to the variation tendency of the lncRNA expression and avoid noise. We set different threshold values to obtain DSS matrixes and found the similarity among these matrixes. The tendency consisted with our previous work [28]. It was suggested that fine-tuned parameters did not cause the remarkable influences. We selected different DSS thresholds for lncRNA classification and performed functional enrichment with DAVID. Notably, the lncRNAs with similar DSS involved in the same functions, while others played different roles in cancer biology. The downside of the analysis was that the lncRNAs with different dosage sensitivity may target the same PCGs. Therefore, we could not distinguish the function of IncRNA with DSS thresholds directly. Further functional validation of the lncRNAs with different DSS can be confirmed by experiment.

Accumulated evidence pointed out that the deregulation of lncRNAs was one of the driving factors for cancer [40, 73-75]. The two lncRNAs, ENSG00000259974 (LINC00261) and ENSG00000268388 that occurred copy number delection were down-regulated in our study. White et al. indicated that the two lncRNAs were associated with lung cancer [76]. In order to figure out the functions of SCNA IncRNAs in LUAD, we performed strict approach to analyze the lncRNAs based on lncRNAs-neighboring PCGs interactions and lncRNA-TF-PCG triples. Published medical literature showed that these lncRNAs were associated with the development and progression of lung cancer. For example, ENSG00000225969 could regulate the expression of CLDN4 by cis-regulation and lead to the carcinogenesis of LUAD. Further, we matched these lncRNAs to specific functions by cancer markers and screened out candidate targets that related with immunoreaction. These results may provide clues for LUAD immunotherapy.

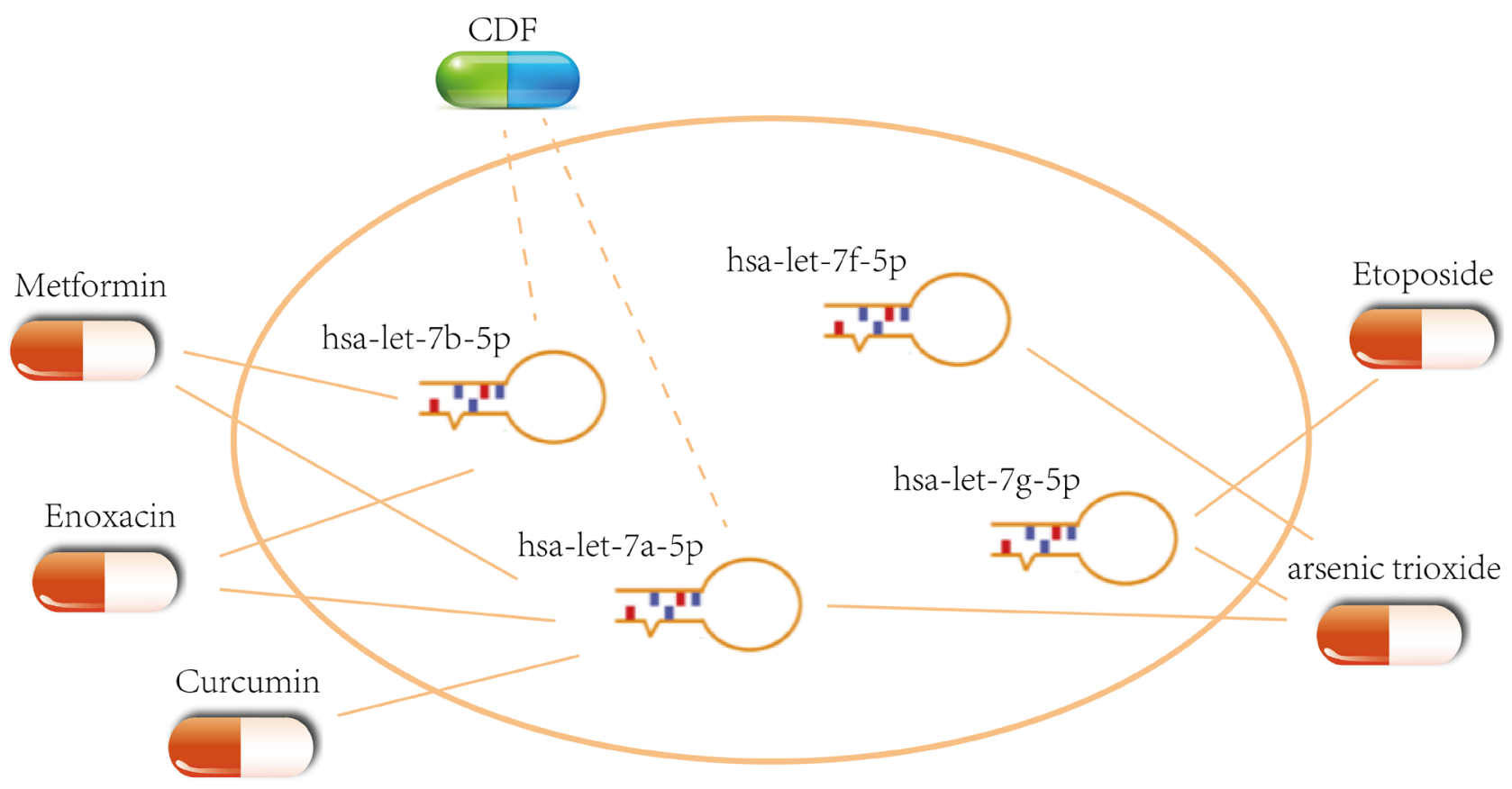

Potential drug to treat LUAD

Experimental validation drug to treat LUAD

Figure 10: Potential small molecule drugs for LUAD treatment. The capsules represent the experimental validation drugs and potential drug respectively. 
We used strict approach to construct ceRNA network. The approach not only considered the lncRNAs/ PCGs that acted as miRNA sponges, but also selected negative miRNA-target interactions. In addition, we provided the potential small molecule drugs that regulated the ceRNA network. Some small molecule drugs, such as Enoxacin and Etoposide, regulated the competitive relations of lncRNA-PCG pairs by influencing the expression of hsa-let-7a/b/f/g-5p, which provided novel clues for LUAD treatment.

Previous study solely focused on the analysis of single lncRNA and lacked systematic research. In order to analyze the function of lncRNAs in 3 regulatory network, we constructed lncRNA-function network and mapped each lncRNA to matched GO terms. This results increased the understanding of lncRNAs and provided reference for functional research. LncRNAs could serve as cancer biomarker because of their tissue specificity [77]. Combining the expression of functional lncRNAs with the clinic information of LUAD patients, we identified prognosis-related ceRNA pairs. In conclusion, these results will help understand the development and progression of SCNA lncRNAs in cancer and provide deeply insights into the cancer biological mechanism.

Similar to other computational approaches, our study also had some limitations. First, most of the lncRNAs that mapped to the copy number delection regions did not have expression value or have low expression in LUAD, leading to the missing of SCNA lncRNAs. Second, we used cancer marker-related GO terms to construct lncRNA-function network, while these GO terms only represented a small fraction. Last, for survival analysis, because of lacking other clinic data that matched with ours, it was hard to further validate prognosis-related lncRNA. These deficiencies would be improved by the complement of data in future.

\section{MATERIALS AND METHODS}

\section{Data and pre-processing}

The RNA-seqV2 data, SCNA profile and clinic information of LUAD were downloaded from The Cancer Genome Atlas (TCGA). The RSEM values of PCGs were normalized by upper quartile. We obtained an expression matrix that contained 576 patient samples (59 normal samples and 517 tumor samples) and 20,513 PCGs by combining the RNA-seqV2 data. PCGs with missing values in $>30 \%$ of the samples were removed. In total, we obtained 15,149 PCGs. Moreover, we added 2 to the expression value of each PCG and performed $\log 2$ transformed. The lncRNA profile of LUAD was obtained from TANRIC database. The lncRNAs that either 50\% quantile $=0$ or $90 \%$ quantile $\leq 0.1$ were removed. We added 0.05 to the expression value of each lncRNA and performed log2-transformed. Finally, we obtained 5,109 lncRNAs and 546 patient samples (58 normal samples). The hg19 (GRCh37) comprehensive gene annotation and lncRNA annotation were downloaded from GENCODE database.

\section{Combining linear and nonlinear regression to calculate dosage sensitive score}

First, we considered the samples with SCNA values in $[-0.3,0.3]$ as normal ones, which meant that the samples had no SCNA. Second, in order to summary the tendency between IncRNA expression and SCNAs, the lncRNAs that had SCNA in more than $20 \%$ samples were selected for further analysis. Considering SCNA value and expression value as variable, we evaluated the effect of SCNA on lncRNAs. The method of calculating DSS [28] that we selected took more information into consideration and was more accurate than the method that based on samples. The higher score of the DSS of PCGs or lncRNAs was, the stronger their dosage sensitivity was.

\section{DNA methylation}

DNA methylation data of LUAD from Illumina Infinium Human Methylation 450 Beadchip (Infinium 450k) arrays was obtained from TCGA, including 485577 probes, 475 tumor samples and 32 normal samples. We then mapped cg probes to promoter regions. The promoter regions were defined as the regions $2 \mathrm{~kb}$ upstream from the TSSs [78]. The probes that mapped to more than one promoter regions were removed. The missing values were replaced by average values. If multiple probes were mapped to the same lncRNA, the average value was considered as the methylation beta value of the lncRNA. The $t$-test was performed to identify the differential methylation genes (fold change $>2$, FDR $<0.01$ ). The fold change value of each gene was calculated by the ratio of the average methylation beta value of tumor samples and normal samples.

\section{Differential expression analysis}

We performed SAM algorithm to identify differentially expressed genes based on PCG profile and lncRNA profile. For each PCG or lncRNA, the R samr package [79] was used for selecting differentially expressed genes (fold change $>2$ ). The BenjaminiHochberg (BH) method was used to calculate FDR value (FDR $<0.05$ ). In total, we obtained 3,654 differentially expressed PCGs (2,185 down-regulated PCGs and 1,469 up-regulated PCGs) and 608 differentially expressed lncRNAs (382 down-regulated lncRNAs and 426 upregulated lncRNAs). 


\section{LncRNA-PCG co-expression}

The Pearson Correlation Coefficient (PCC) was used to calculate the co-expressed coefficient between SCNA lncRNAs and differentially expressed PCGs (BH method, FDR $<0.05, r>0.5$ ).

\section{LncRNA-miRNA interaction pairs}

The IncRNA-miRNA and mRNA-miRNA interaction pairs were obtained from starBase v2.0. After removing redundancy, we obtained 10,112 lncRNAmiRNA interaction pairs, including 132 miRNAs and 1,114 lncRNAs, and 323,648 mRNA-miRNA interaction pairs, including 366 miRNAs and 12,386 mRNAs.

\section{Cis-acting and trans-acting regulation prediction}

The expression profiles of $\operatorname{lncRNAs}$ and PCGs were used to predict the cis-acting regulation and trans-acting regulation in our study [33, 34]. For cis-acting regulation, we recognized coupled genomic locations of lncRNAs and PCGs, the neighboring genes within $300 \mathrm{~kb}$ of the lncRNAs were considered as potential cis-acting targets of lncRNAs.

LncRNAs may function via TFs in trans-acting regulation. First, we obtained experimentally verified TF-gene interaction pairs from TRED. Second, PCC value was used to select the TFs that co-expressed with SCNA lncRNA (FDR $<0.05, r>0.6$ ). Finally, for each given IncRNA, we calculated the overlaps between coexpressed PCGs and the target genes of a given TF. The hypergeometric distribution method was performed to test the significance of the overlaps. If the co-expressed PCGs of a given lncRNA were significantly overlapped with the target genes of a given TF, the TF may interact with the lncRNA, and these PCGs may be regarded as the transacting target of the lncRNA.

\section{The construction of ceRNA-ceRNA network}

The intersection of co-expression lncRNA-PCG pairs and lncRNA-miRNA interaction pairs was used to identify ceRNA pairs. A hypergeometric test was performed to evaluate the significance of shared miRNAs for each possible gene pair:

$$
P=1-\sum_{i=0}^{x-1} \frac{\left(\begin{array}{l}
\mathrm{L} \\
\mathrm{i}
\end{array}\right)\left(\begin{array}{l}
\mathrm{N}-\mathrm{L} \\
\mathrm{M}-\mathrm{i}
\end{array}\right)}{\left(\begin{array}{l}
\mathrm{N} \\
\mathrm{M}
\end{array}\right)}
$$

Where $\mathrm{N}$ is the total number of miRNAs which were interacted with lncRNAs or PCGs, $\mathrm{M}$ is the number of miRNAs interacting with this given lncRNA, $\mathrm{L}$ is the number of miRNAs interacting with this given PCGs, and $\mathrm{x}$ is the number of miRNAs that interact with both of them, respectively. The P-value and FDR correction less than 0.05 were used as the threshold. Finally, the significant ceRNA pairs were used to construct ceRNAceRNA network. We used Cytoscape v3.1 to visualize the network and analyze the topological property of network.

\section{Abbreviations}

SCNA: somatic copy number alternation; DSS: dosage sensitivity score; LUAD: lung adenocarcinoma; NSCLC: non-small cell lung cancer; PCGs: proteincoding genes.

\section{Authors' contributions}

YX designed the study, YW and ZY performed the research and wrote the manuscript. YW, CW, ZY, QZ, $\mathrm{KL}$ and $\mathrm{YZ}$ analyzed the data. All authors reviewed the manuscript.

\section{CONFLICTS OF INTEREST}

The authors declared that they have no conflicts of interest.

\section{FUNDING}

The National Natural Science Foundation of China (grant numbers 81372492 and 81673036).

\section{REFERENCES}

1. Chen J, Zhang K, Song H, Wang R, Chu X, Chen L. Long noncoding RNA CCAT1 acts as an oncogene and promotes chemoresistance in docetaxel-resistant lung adenocarcinoma cells. Oncotarget. 2016; 7:62474-89. https://doi.org/10.18632/oncotarget.11518.

2. Gutschner T, Hämmerle M, Eissmann M, Hsu J, Kim Y, Hung G, Revenko A, Arun G, Stentrup M, Gross M, Zörnig M, MacLeod AR, Spector DL, Diederichs S. The noncoding RNA MALAT1 is a critical regulator of the metastasis phenotype of lung cancer cells. Cancer Res. 2013; 73: 11801189.

3. Imielinski M, Berger AH, Hammerman PS, Hernandez B, Pugh TJ, Hodis E, Cho J, Suh J, Capelletti M, Sivachenko A, Sougnez C, Auclair D, Lawrence MS, et al. Mapping the hallmarks of lung adenocarcinoma with massively parallel sequencing. Cell. 2012; 150: 1107-1120.

4. Mercer TR, Dinger ME, Mattick JS. Long non-coding RNAs: insights into functions. Nat Rev Genet. 2009; 10: 155-159.

5. Huarte M, Guttman M, Feldser D, Garber M, Koziol MJ, 
Kenzelmann-Broz D, Khalil AM, Zuk O, Amit I, Rabani M, Attardi LD, Regev A, Lander ES, et al. A large intergenic noncoding RNA induced by p53 mediates global gene repression in the p53 response. Cell. 2010; 142: 409-419.

6. Dimitrova N, Zamudio JR, Jong RM, Soukup D, Resnick R, Sarma K, Ward AJ, Raj A, Lee JT, Sharp PA, Jacks T. LincRNA-p21 activates p21 in cis to promote Polycomb target gene expression and to enforce the G1/S checkpoint. Mol Cell. 2014; 54: 777-790.

7. Nancarrow DJ, Handoko HY, Smithers BM, Gotley DC, Drew PA, Watson DI, Clouston AD, Hayward NK, Whiteman DC. Genome-wide copy number analysis in esophageal adenocarcinoma using high-density singlenucleotide polymorphism arrays. Cancer Res. 2008; 68: 4163-4172.

8. Stephens PJ, Tarpey PS, Davies H, Van Loo P, Greenman C, Wedge DC, Nik-Zainal S, Martin S, Varela I, Bignell GR, Yates LR, Papaemmanuil E, Beare D, et al. The landscape of cancer genes and mutational processes in breast cancer. Nature. 2012; 486: 400-404.

9. Guichard C, Amaddeo G, Imbeaud S, Ladeiro Y, Pelletier L, Maad IB, Calderaro J, Bioulac-Sage P, Letexier M, Degos F, Clement B, Balabaud C, Chevet E, et al. Integrated analysis of somatic mutations and focal copynumber changes identifies key genes and pathways in hepatocellular carcinoma. Nat Genet. 2012; 44: 694-698.

10. Campbell JD, Alexandrov A, Kim J, Wala J, Berger AH, Pedamallu CS, Shukla SA, Guo G, Brooks AN, Murray BA, Imielinski M, Hu X, Ling S, et al. Distinct patterns of somatic genome alterations in lung adenocarcinomas and squamous cell carcinomas. Nat Genet. 2016; 48: 607-616.

11. Solvang HK, Lingjaerde OC, Frigessi A, Borresen-Dale AL, Kristensen VN. Linear and non-linear dependencies between copy number aberrations and mRNA expression reveal distinct molecular pathways in breast cancer. BMC Bioinformatics. 2011; 12: 197.

12. Liu Y, Zhang R, Zhao N, Zhang Q, Yan Z, Chang Z, Wei $\mathrm{Y}, \mathrm{Wu} \mathrm{C}, \mathrm{Xu} \mathrm{J}, \mathrm{Xu} \mathrm{Y}$. A comparative analysis reveals the dosage sensitivity and regulatory patterns of lncRNA in prostate cancer. Mol Biosyst. 2016; 12: 3176-3185.

13. Yan X, Hu Z, Feng Y, Hu X, Yuan J, Zhao SD, Zhang Y, Yang L, Shan W, He Q, Fan L, Kandalaft LE, Tanyi JL, et al. Comprehensive Genomic Characterization of Long Noncoding RNAs across Human Cancers. Cancer Cell. 2015; 28: 529-540.

14. Tseng YY, Moriarity BS, Gong W, Akiyama R, Tiwari A, Kawakami H, Ronning P, Reuland B, Guenther K, Beadnell TC, Essig J, Otto GM, O'Sullivan MG, et al. PVT1 dependence in cancer with MYC copy-number increase. Nature. 2014; 512: 82-86.

15. Gabory A, Jammes H, Dandolo L. The H19 locus: role of an imprinted non-coding RNA in growth and development. BioEssays. 2010; 32: 473-480.

16. Guo X, Gao L, Liao Q, Xiao H, Ma X, Yang X, Luo H,
Zhao G, Bu D, Jiao F, Shao Q, Chen R, Zhao Y. Long non-coding RNAs function annotation: a global prediction method based on bi-colored networks. Nucleic Acids Res. 2013; 41: e35.

17. Cabili MN, Trapnell C, Goff L, Koziol M, Tazon-Vega B, Regev A, Rinn JL. Integrative annotation of human large intergenic noncoding RNAs reveals global properties and specific subclasses. Genes Dev. 2011; 25: 1915-1927.

18. Quinn JJ, Chang HY. Unique features of long non-coding RNA biogenesis and function. Nat Rev Genet. 2016; 17: 47-62.

19. Ulitsky I, Bartel DP. lincRNAs: genomics, evolution, and mechanisms. Cell. 2013; 154: 26-46.

20. Pasut A, Matsumoto A, Clohessy JG, Pandolfi PP. The pleiotropic role of non-coding genes in development and cancer. Curr Opin Cell Biol. 2016; 43: 104-113.

21. Guttman M, Amit I, Garber M, French C, Lin MF, Feldser D, Huarte M, Zuk O, Carey BW, Cassady JP, Cabili MN, Jaenisch R, Mikkelsen TS, et al. Chromatin signature reveals over a thousand highly conserved large non-coding RNAs in mammals. Nature. 2009; 458: 223-227.

22. Tian D, Sun S, Lee JT. The long noncoding RNA, Jpx, is a molecular switch for X chromosome inactivation. Cell. 2010; 143: 390-403.

23. Wang KC, Chang HY. Molecular mechanisms of long noncoding RNAs. Mol Cell. 2011; 43: 904-914.

24. Bond AM, Vangompel MJ, Sametsky EA, Clark MF, Savage JC, Disterhoft JF, Kohtz JD. Balanced gene regulation by an embryonic brain ncRNA is critical for adult hippocampal GABA circuitry. Nat Neurosci. 2009; 12: 1020-1027.

25. Salmena L, Poliseno L, Tay Y, Kats L, Pandolfi PP. A ceRNA hypothesis: the Rosetta Stone of a hidden RNA language? Cell. 2011; 146: 353-358.

26. Xu J, Li Y, Lu J, Pan T, Ding N, Wang Z, Shao T, Zhang J, Wang L, Li X. The mRNA related ceRNA-ceRNA landscape and significance across 20 major cancer types. Nucleic Acids Res. 2015; 43: 8169-8182.

27. Wang ZL, Li B, Piccolo SR, Zhang XQ, Li JH, Zhou H, Yang JH, Qu LH. Integrative analysis reveals clinical phenotypes and oncogenic potentials of long non-coding RNAs across 15 cancer types. Oncotarget. 2016; 7:3504455. https://doi.org/10.18632/oncotarget.9037.

28. Yan Z, Liu Y, Wei Y, Zhao N, Zhang Q, Wu C, Chang Z, $\mathrm{Xu} Y$. The functional consequences and prognostic value of dosage sensitivity in ovarian cancer. Mol Biosyst. 2017; 13: 380-391.

29. Anagnostou VK, Brahmer JR. Cancer immunotherapy: a future paradigm shift in the treatment of non-small cell lung cancer. Clin Cancer Res. 2015; 21: 976-984.

30. Brahmer JR, Pardoll DM. Immune checkpoint inhibitors: making immunotherapy a reality for the treatment of lung cancer. Cancer Immunol Res. 2013; 1: 85-91.

31. Sherr CJ, Bartek J. Cell Cycle-Targeted Cancer Therapies. 
Annual Review of Cancer Biology. 2017; 1:41-57.

32. Tang W, Liao Z, Zou Q. Which statistical significance test best detects oncomiRNAs in cancer tissues? An exploratory analysis. Oncotarget. 2016; 7:85613-23. https://doi. org/10.18632/oncotarget.12828.

33. Xiong W, Jiang YX, Ai YQ, Liu S, Wu XR, Cui JG, Qin JY, Liu Y, Xia YX, Ju YH, He WJ, Wang Y, Li YF, et al. Microarray Analysis of Long Non-coding RNA Expression Profile Associated with 5-Fluorouracil-Based Chemoradiation Resistance in Colorectal Cancer Cells. Asian Pac J Cancer Prev. 2015; 16: 3395-3402.

34. Vance KW, Sansom SN, Lee S, Chalei V, Kong L, Cooper SE, Oliver PL, Ponting CP. The long non-coding RNA Paupar regulates the expression of both local and distal genes. EMBO J. 2014; 33: 296-311.

35. Noyola DE, Alarcon A, Noguera-Julian A, Muntasell A, Munoz-Almagro C, Garcia J, Mur A, Fortuny C, LopezBotet M. Dynamics of the NK-cell subset redistribution induced by cytomegalovirus infection in preterm infants. Hum Immunol. 2015; 76: 118-123.

36. Liu X, Yu X, Xie J, Zhan M, Yu Z, Xie L, Zeng H, Zhang F, Chen G, Yi X, Zheng J. ANGPTL2/LILRB2 signaling promotes the propagation of lung cancer cells. Oncotarget. 2015; 6:21004-15. https://doi.org/10.18632/ oncotarget. 4217.

37. Spits H, Cupedo T. Innate lymphoid cells: emerging insights in development, lineage relationships, and function. Annu Rev Immunol. 2012; 30: 647-675.

38. Bruno A, Focaccetti C, Pagani A, Imperatori AS, Spagnoletti M, Rotolo N, Cantelmo AR, Franzi F, Capella C, Ferlazzo G, Mortara L, Albini A, Noonan DM. The proangiogenic phenotype of natural killer cells in patients with non-small cell lung cancer. Neoplasia. 2013; 15: 133 142.

39. Kim N, Takami M, Rho J, Josien R, Choi Y. A novel member of the leukocyte receptor complex regulates osteoclast differentiation. J Exp Med. 2002; 195: 201-209.

40. Ashouri A, Sayin VI, Van den Eynden J, Singh SX, Papagiannakopoulos T, Larsson E. Pan-cancer transcriptomic analysis associates long non-coding RNAs with key mutational driver events. Nat Commun. 2016; 10: 13197.

41. Fafilek B, Krausova M, Vojtechova M, Pospichalova V, Tumova L, Sloncova E, Huranova M, Stancikova J, Hlavata A, Svec J, Sedlacek R, Luksan O, Oliverius M, et al. Troy, a tumor necrosis factor receptor family member, interacts with $\operatorname{lgr} 5$ to inhibit wnt signaling in intestinal stem cells. Gastroenterology. 2013; 144: 381-391.

42. Stancikova J, Krausova M, Kolar M, Fafilek B, Svec J, Sedlacek R, Neroldova M, Dobes J, Horazna M, Janeckova L, Vojtechova M, Oliverius M, Jirsa M, et al. NKD1 marks intestinal and liver tumors linked to aberrant Wnt signaling. Cell Signal. 2015; 27: 245-256.

43. Zhang S, Wang Y, Dai SD, Wang EH. Down-regulation of NKD1 increases the invasive potential of non-small-cell lung cancer and correlates with a poor prognosis. BMC Cancer. 2011; 11: 186.

44. Tang Y, Shu G, Yuan X, Jing N, Song J. FOXA2 functions as a suppressor of tumor metastasis by inhibition of epithelial-to-mesenchymal transition in human lung cancers. Cell Res. 2011; 21: 316-326.

45. Li CM, Gocheva V, Oudin MJ, Bhutkar A, Wang SY, Date SR, Ng SR, Whittaker CA, Bronson RT, Snyder EL, Gertler FB, Jacks T. Foxa2 and Cdx2 cooperate with Nkx2-1 to inhibit lung adenocarcinoma metastasis. Genes Dev. 2015; 29: 1850-1862.

46. Kaczkowski B, Tanaka Y, Kawaji H, Sandelin A, Andersson R, Itoh M, Lassmann T, Hayashizaki Y, Carninci P, Forrest AR. Transcriptome Analysis of Recurrently Deregulated Genes across Multiple Cancers Identifies New Pan-Cancer Biomarkers. Cancer Res. 2016; 76: 216-226.

47. Yamada G, Murata M, Takasawa A, Nojima M, Mori Y, Sawada N, Takahashi H. Increased expressions of claudin 4 and 7 in atypical adenomatous hyperplasia and adenocarcinoma of the lung. Med Mol Morphol. 2016; 49: 163-169.

48. Yamaguchi E, Nakayama T, Nanashima A, Matsumoto K, Yasutake T, Sekine I, Nagayasu T. Ets-1 proto-oncogene as a potential predictor for poor prognosis of lung adenocarcinoma. Tohoku J Exp Med. 2007; 213: 41-50.

49. Hung JJ, Hsueh CT, Chen KH, Hsu WH, Wu YC. Clinical significance of E2F1 protein expression in non-small cell lung cancer. Exp Hematol Oncol. 2012; 1: 18.

50. Khirade MF, Lal G, Bapat SA. Derivation of a fifteen gene prognostic panel for six cancers. Sci Rep. 2015; 5: 13248.

51. Fu HY, Li C, Yang W, Gai XD, Jia T, Lei YM, Li Y. FOXP3 and TLR4 protein expression are correlated in nonsmall cell lung cancer: implications for tumor progression and escape. Acta Histochem. 2013; 115: 151-157.

52. Zeng X, Zhang X, Zou Q. Integrative approaches for predicting microRNA function and prioritizing diseaserelated microRNA using biological interaction networks. Brief Bioinform. 2016; 17: 193-203.

53. Liu Y, Zeng X, He Z, Zou Q. Inferring microRNA-disease associations by random walk on a heterogeneous network with multiple data sources.IEEE/ACM Trans Comput Biol Bioinform. 2017; 14: 905-15.

54. Zou Q, Li J, Hong Q, Lin Z, Wu Y, Shi H, Ju Y. Prediction of MicroRNA-Disease Associations Based on Social Network Analysis Methods. Biomed Res Int. 2015; 2015: 810514.

55. Liu D, Yu X, Wang S, Dai E, Jiang L, Wang J, Yang Q, Yang F, Zhou S, Jiang W. The gain and loss of long noncoding RNA associated-competing endogenous RNAs in prostate cancer. Oncotarget. 2016; 7:57228-38. https:// doi.org/10.18632/oncotarget.11128.

56. Hu J, Yan J, Rao G, Latha K, Overwijk WW, Heimberger $\mathrm{AB}, \mathrm{Li}$ S. The Duality of Fgl2 - Secreted Immune 
Checkpoint Regulator Versus Membrane-Associated Procoagulant: Therapeutic Potential and Implications. Int Rev Immunol. 2016; 35: 325-339.

57. Wang J, Zhang Y, Wan H, Dong N, Bao L, Sun X, Xu M, Wang X. Global analyses of subtype- or stage-specific genes on chromosome 7 in patients with lung cancer. Cancer Metastasis Rev. 2015; 34: 333-345.

58. Yuan H, Liu H, Liu Z, Owzar K, Han Y, Su L, Wei Y, Hung RJ, McLaughlin J, Brhane Y, Brennan P, Bickeboeller H, Rosenberger A, et al. A Novel Genetic Variant in Long Non-coding RNA Gene NEXN-AS1 is Associated with Risk of Lung Cancer. Sci Rep. 2016; 6: 34234.

59. Li Y, Xu J, Chen H, Bai J, Li S, Zhao Z, Shao T, Jiang $\mathrm{T}$, Ren H, Kang C, Li X. Comprehensive analysis of the functional microRNA-mRNA regulatory network identifies miRNA signatures associated with glioma malignant progression. Nucleic Acids Res. 2013; 41: e203.

60. Richards EJ, Permuth-Wey J, Li Y, Chen YA, Coppola D, Reid BM, Lin HY, Teer JK, Berchuck A, Birrer MJ, Lawrenson K, Monteiro AN, Schildkraut JM, et al. A functional variant in HOXA11-AS, a novel long noncoding RNA, inhibits the oncogenic phenotype of epithelial ovarian cancer. Oncotarget. 2015; 6:34745-57. https://doi. org/10.18632/oncotarget.5784.

61. Liang J, Lv J, Liu Z. Identification of stage-specific biomarkers in lung adenocarcinoma based on RNA-seq data. Tumour Biol. 2015; 36: 6391-6399.

62. Le TD, Zhang J, Liu L, Li J. Computational methods for identifying miRNA sponge interactions. Brief Bioinform. 2017; 18:577-90.

63. Tay Y, Rinn J, Pandolfi PP. The multilayered complexity of ceRNA crosstalk and competition. Nature. 2014; 505: 344-352.

64. Zhang Y, He RQ, Dang YW, Zhang XL, Wang X, Huang SN, Huang WT, Jiang MT, Gan XN, Xie Y, Li P, Luo DZ, Chen G, et al. Comprehensive analysis of the long noncoding RNA HOXA11-AS gene interaction regulatory network in NSCLC cells. Cancer Cell Int. 2016; 16: 89.

65. Meng F, Wang J, Dai E, Yang F, Chen X, Wang S, Yu X, Liu D, Jiang W. Psmir: a database of potential associations between small molecules and miRNAs. Sci Rep. 2016; 6: 19264.

66. Wang J, Meng F, Dai E, Yang F, Wang S, Chen X, Yang L, Wang Y, Jiang W. Identification of associations between small molecule drugs and miRNAs based on functional similarity. Oncotarget. 2016; 7:38658-69. https://doi. org/10.18632/oncotarget.9577.

67. Liu X, Wang S, Meng F, Wang J, Zhang Y, Dai E, Yu X, Li X, Jiang W. SM2miR: a database of the experimentally validated small molecules' effects on microRNA expression. Bioinformatics. 2013; 29: 409-411.
68. Wu SH, Hang LW, Yang JS, Chen HY, Lin HY, Chiang JH, Lu CC, Yang JL, Lai TY, Ko YC, Chung JG. Curcumin induces apoptosis in human non-small cell lung cancer NCI-H460 cells through ER stress and caspase cascade- and mitochondria-dependent pathways. Anticancer Res. 2010; 30: 2125-2133.

69. Lin SS, Lai KC, Hsu SC, Yang JS, Kuo CL, Lin JP, Ma YS, Wu CC, Chung JG. Curcumin inhibits the migration and invasion of human A549 lung cancer cells through the inhibition of matrix metalloproteinase-2 and -9 and Vascular Endothelial Growth Factor (VEGF). Cancer Lett. 2009; 285: 127-133.

70. Chen HW, Lee JY, Huang JY, Wang CC, Chen WJ, Su SF, Huang CW, Ho CC, Chen JJ, Tsai MF, Yu SL, Yang PC. Curcumin inhibits lung cancer cell invasion and metastasis through the tumor suppressor HLJ1. Cancer Res. 2008; 68: 7428-7438.

71. Wang J, Gao Q, Wang D, Wang Z, Hu C. Metformin inhibits growth of lung adenocarcinoma cells by inducing apoptosis via the mitochondria-mediated pathway. Oncol Lett. 2015; 10: 1343-1349.

72. Lin JJ, Gallagher EJ, Sigel K, Mhango G, Galsky MD, Smith CB, LeRoith D, Wisnivesky JP. Survival of patients with stage IV lung cancer with diabetes treated with metformin. Am J Respir Crit Care Med. 2015; 191: 448454.

73. Ji P, Diederichs S, Wang W, Boing S, Metzger R, Schneider PM, Tidow N, Brandt B, Buerger H, Bulk E, Thomas M, Berdel WE, Serve H, et al. MALAT-1, a novel noncoding RNA, and thymosin beta4 predict metastasis and survival in early-stage non-small cell lung cancer. Oncogene. 2003; 22: 8031-8041.

74. Prensner JR, Chinnaiyan AM. The emergence of lncRNAs in cancer biology. Cancer Discov. 2011; 1: 391-407.

75. Esteller M. Non-coding RNAs in human disease. Nat Rev Genet. 2011; 12: 861-874.

76. White NM, Cabanski CR, Silva-Fisher JM, Dang HX, Govindan R, Maher CA. Transcriptome sequencing reveals altered long intergenic non-coding RNAs in lung cancer. Genome Biol. 2014; 15: 429.

77. Schmitt AM, Chang HY. Long Noncoding RNAs in Cancer Pathways. Cancer Cell. 2016; 29: 452-463.

78. Wang Z, Zang C, Rosenfeld JA, Schones DE, Barski A, Cuddapah S, Cui K, Roh TY, Peng W, Zhang MQ, Zhao $\mathrm{K}$. Combinatorial patterns of histone acetylations and methylations in the human genome. Nat Genet. 2008; 40: 897-903.

79. Tusher VG, Tibshirani R, Chu G. Significance analysis of microarrays applied to the ionizing radiation response. Proc Natl Acad Sci USA. 2001; 98:5116-21. 